\title{
Slit2-Robo1 signaling enhances nasopharyngeal carcinoma metastasis via PI3K/AKT/ $\beta$-catenin pathway
}

Mei Shi ( $\sim$ mshi82@hotmail.com )

Xijing Hospital

Li-Na Zhao

Xijing Hospital

Rui Ma

Xijing Hospital

Hua Yang

Xijing Hospital

Chang-Hao Liu

Xijing Hospital

Jing Hu

Xijing Hospital

\section{Research}

Keywords: Nasopharyngeal carcinoma, Metastasis, Slit2, Robo1, PI3K/AKT/ $\beta$-catenin.

Posted Date: June 11th, 2020

DOl: https://doi.org/10.21203/rs.3.rs-34575/v1

License: (1) This work is licensed under a Creative Commons Attribution 4.0 International License. Read Full License 


\section{Abstract}

Background Distant metastasis remained the major cause of mortality in nasopharyngeal carcinoma (NPC) receiving radiotherapy despite favorable locoregional control in the past decades. Tumor microenvironment plays a critical role in tumor progression by secreting factors that regulate cancer cell metastasis. We explored the role of secreted protein Slit2 in NPC metastasis and the underlying mechanisms.

Methods Colony formation assay were determined the opitimal dose. Using real-time PCR, western blotting, and ELISA, we detected the expression of Slit2 in NPC cells and in supernatant. Cell migration assay and cell invasion assay were examined the role of Slit2 in promoting metastasis of NPC cell lines. Western blotting, immunofluorescence staining, transwell and Co-IP assay et al. were performed to explore the detailed molecular mechanism of Slit2 in NPC. Finally, we estimated the effects on Slit2shRNA NPC cells in vivo tumor metastasis experiment

Results We found that irradiated dying NPC cells could secrete Slit2 to enhance the metastatic ability of surviving NPC cells. Mechanistic study showed that Slit2 interacted with its cognate Robo1 receptor, which activated PI3K/Akt pathway and enhanced the nuclear translocation of $\beta$-catenin to promote NPC metastasis. Finally, we demonstranted that knockdown of Slit2 in NPC cells significantly suppressed lung and liver metastasis.

Conclusion Slit2, a secreted factor released from irradiated dying NPC cells could promote metastasis of living/resistent NPC cells through PI3K/AKT/ $\beta$-catenin pathway. Tumor microenvironment altered by irradiated dying cancer cells may be promising target to treat cancer metastasis.

\section{Background}

Nasopharyngeal carcinoma (NPC) is the most common head and neck cancer in Southeast Asia. The current standard of care for NPC is radiotherapy alone for early stage NPC or concurrent chemoradiation (CCRT) for locally advanced NPC[1]. However, despite a high 5-year local control rate for locally advanced NPC receiving definite radiotherapy based treatment, distant metastasis remains the predominant pattern of failure demonstrated by our[2] and other studies[3,4]. To date, few molecules have been found to be correlated with NPC metastasis after definite radiotherapy. However, the mechanism of NPC metastasis is too complicated after radiotheapy and radiotherapy related specific tumor microenvironment might be helpful to elucidate the underlying mechanisms.

Previous study indicated that the metastatic ability was significantly higher for tumors in preirradiated beds than those in non-irradiated beds [5], indicating " tumor bed effect " could promote tumor metastasis by changing tumor microenvironment [6]. Several studies have reported that irradiation may promote the invasion ability of the living cancer cells after irradiation[7]. Our previous study showed that ionizing radiation could promote migration and invasion of cancer cells through transforming growth factor-beta-mediated epithelial-mesenchymal transition [8]. Huang et al also reported that dying mouse 
breast tumor cells treated by radiotherapy could produce growth-stimulating signals to stimulate the repopulation and proliferation of surviving tumor cells through Caspase3-PGE2 pathway [9]. However, whether radiotherapy remodeled microenvironment could contribute to NPC metastasis and which molecules are critically involved in this processs remained unclear.

Here we tested the hypothesis that necrotic or apoptotic NPC cells killed by radiotherapy could release metastasis-stimulating signals to the microenvironment and promote the metastatic ability of the surviving cancer cells. Distant metastases was partially due to the enhanced invasiveness properties of the surviving cancer cells resistant to radiotherapy. Our previous study showed that Slit2-Robo1 interaction may contribute to gastric cancer metastasis[10], but whether Slit2 was involved in NPC metastasis was unknown. We for the first time constructed a co-culture system and found that the supernatant of lethally irradiated NPC CNE1 cell line could promote migration and invasion of radioresistant NPC cell line CNE1-IR. We further provided evidence that Slit2 secreted from irradiated dying NPC cells could promote metastasis of living/resistent NPC cells through PI3K/AKT/ $\beta$-catenin pathway.

\section{Methods}

\section{Cell lines}

The NPC cell line, CNE1, was obtained from the Shanghai Institutes for Biological Sciences (Chinese Academy of Sciences, Shanghai, China). CNE1 cells were irradiated with X-ray doses of 2 Gy every other day for 40 days, resulting in 20 total irradiation treatments. Surviving cells were considered radioresistant (CNE1/IR) and were cultured in RPMI-1640 (Gibco; Invitrogen, Carlsbad, California, USA) supplemented with $10 \%$ FBS (HyClone, Thermo, USA) and $1 \%$ penicillin-streptomycin in a humidified incubator at $37^{\circ} \mathrm{C}$ and 5\% C02.Slit2, Robo1, Robo2 shRNA and scrambled control shRNA carrying lentivirus were purchased from Gene Pharma. CNE1-IR/NC, CNE1-IR/Slit2, CNE1-IR/Robo1 or CNE1-IR/Robo2 were produced by infecting CNE1-IR cells with Lentiviral vectors LV3-NC, LV3-shSlit2, LV3-Robo1 and LV3-Robo2, separately.

\section{Colony formation assay}

The colony formation assay was employed to confirm a lethal dose of NPC cells with IR. CNE1 cells were plated onto 6-well plates after IR. Cells were exposed to radiation in a Gamma Cell Radiator (RS-2000 XE Biological Irradiator, RAD. SOURCE) using $X$-rays as a radioactive source. A radiation dose of $1.47 \mathrm{~Gy} / \mathrm{min}$ was applied to all groups., and after administration of the different doses 0Gy, 4Gy, 8Gy, 12Gy, 16Gy, 20Gy. The medium was changed after irradiation for 24 hours. After two weeks, cell colonies were fixed with methanol, stained with $0.1 \%$ crystal violet, and scored by counting the number of colonies with an inverted microscope, using the standard definition that a colony consists of 50 or more cells.

\section{Enzyme-linked immunosorbent assay (ELISA)}


The levels of Slit2 were detected by ELISA (Bio-swamp) in supernatural of lethal CNE1 cells. The samples were incubated in ELISA plates for $90 \mathrm{~min}$ at $37^{\circ} \mathrm{C}$. Then the biotinylated antibodies were added and incubated for $30 \mathrm{~min}$ at $37^{\circ} \mathrm{C}$. After washing with washing buffer, HRP-conjugate reagent were added to each well for $30 \mathrm{~min}$ at $37^{\circ} \mathrm{C}$. The chromogen 3,3',5,5'-tetramethyl benzidine was added to each well to perform enzyme reaction for $15 \mathrm{~min}$ at $37^{\circ} \mathrm{C}$ before washing repeat 5 times. Last, the reactions were stopped by the stop solution. The absorbances were measured at $450 \mathrm{~nm}$ using a microplate reader (BioRad, model no. 680) at $450 \mathrm{~nm}$, and the results were calculated using a calibration curve prepared from standards.

\section{Cell migration assay.}

Cell migration was detected using a Transwell assay. $1 \times 10^{4}$ stable cells in $100 \mu \mathrm{l}$ serum-free medium were added to the top chamber of the transwell $(0.4 \mu \mathrm{m}$ pore size, Corning区USA). The bottom well contained growth medium with the supernatant of lethally irradiated NPC cell lines. Cells were incubated at $37^{\circ} \mathrm{C}$ for $24 \mathrm{~h}$. After $24 \mathrm{~h}$, the cells that up-regulated or knockdown related gene were seeded in the upper chamber. Regular medium containing various agents (rSlit2 and Wortmannin) was added in the lower chamber as the chemoattractant. After incubation at $37^{\circ} \mathrm{C}$ for $12 \mathrm{~h}$, the cells were fixed with ethanol and stained with $0.1 \%$ crystal violet for $30 \mathrm{~min}$. After washing the cells with PBS, the cells on the upper surface of the filter were mechanically removed with a cotton swab. The invading cells on the lower surface were equantified by counting the number of cells that migrated in 10 random microscopic fields per filter at a magnification of $\times 200$ (TE2000,Nikon). These experiments were repeated three times, and three wells were used for each treatment.

\section{Cell invasion assay.}

The invasive ability of cells was confirmed in 24-well transwell chambers (Corning, Corning, NY). The Matrigel was diluted with serum-free medium at the indicated concentrations and then $40 \mu$ of diluted Matrigel was added to the upper chamber, and incubated at $37^{\circ} \mathrm{C}$ for $4 \mathrm{~h}$ to solidify the gel. Cells were implanted and detected as described migration assay. These experiments were repeated three times, and three wells were used for each treatment.

\section{Western blotting.}

At the end of each treatment, the cells were washed twice with ice-cold PBS and then sonicated on ice in a lysis buffer $(50 \mathrm{mM}$ Tris- $\mathrm{HCl}$ pH 7.4, $150 \mathrm{mM} \mathrm{NaCl}, 0.5 \%$ sodium deoxycholate, $1 \%$ Nonidet P-40, $0.1 \%$ SDS, $1 \mathrm{mM} P M S F$, sodium orthovanadate, sodium fluoride and aprotinin). Cell lysates were centrifuged at $12,000 \mathrm{rpm}$ for $10 \mathrm{~min}$ at $4^{\circ} \mathrm{C}$, and the supernatants were collected for western blotting. Protein concentration was measured using a protein assay. After boiling for $5 \mathrm{~min}$ in the loading buffer with $10 \%$ 2-mercaptoethanol, the samples containing $30 \mu \mathrm{g}$ protein were separated by SDS-PAGE and transferred onto nitrocellulose membranes. The membranes were blocked with $5 \%$ non-fat milk-TBST buffer for $1 \mathrm{~h}$ at room temperature and incubated with the corresponding primary antibodies overnight at $4{ }^{\circ} \mathrm{C}$ with gentle agitation. The primary Rabbit antibodies for Slit2, Robo1, Robo2, phosphorylation-AKT, AKT, and $\beta$ - 
catenin were purchased from Cell Signaling Technology, Inc. (Danvers, MA, USA) and were applied at a 1:500 dilution. A 1:1,000 dilution of $\beta$-actin (Cell Signaling Technology, Danvers, MA, USA) were also used as a loading control.The membranes were washed in TBST and incubated for $2 \mathrm{~h}$ with secondary antibodies at room temperature. The signals were detected using ECL reagent and analyzed using Image Lab 4.0 analysis software from Bio-Rad.

\section{Quantitative real-time PCR}

Total RNA from the cultured cells was isolated using the TRIzol reagent according to the manufacturer's instructions. Reverse transcription was carried out with PrimeScript ${ }^{\mathrm{TM}} \mathrm{RT}$ reagent kit according to the standard protocol (Takara, Dalian, China). The primer sequences used were: slit2 Forward:5'CGTTTGGAAAATG TGCAGCATAA-3'Reverse:5'-TTCGATTGCTTCTCAACATCAAAGT-3'; Robo1 Forward:5'GCGTGCAGTACTAAGGGAACA-3'Reverse:5'-GGCTTCTTACATGAACATAATGAA-3';Robo2Forward:5'GGGTTACTACATCTGCCAGGCTT-3'Reverse:5'-AGGTGGAGGTCTATCTGTCAAAACAT-3', qPCR analysis was performed on a Roche LightCycler Nano instrument using FastStart Essential DNA Green Master Mix, and $\beta$-actin Forward: 5' CCTGGGCATGGAGTCCTGTG 3'凶Reverse: 5' TCTTCATTGTGCTG GGTGCC 3' was used as the endogenous control. The qPCR conditions were: pre-incubation at $95^{\circ} \mathrm{C}$ for $10 \mathrm{~min}(1 \mathrm{cycle})$ followed by denaturation of 40 cycles at $95^{\circ} \mathrm{C}$ for $20 \mathrm{sec}$, annealing at $60^{\circ} \mathrm{C}$ for $20 \mathrm{sec}$ and extension at $72^{\circ} \mathrm{C}$ for $20 \mathrm{sec}$. Treatments and conditions were performed in triplicate to calculate the statistical signifcance.

\section{Co-immunoprecipitation Assay}

Robo1 overexpressing CNE1-IR cells were serum starved for $6 \mathrm{~h}$ and treated with rSlit2 for 10,20 and 30 min. Cells were lysed and incubated in lysis buffer $\left(150 \mathrm{mM} \mathrm{NaCl}, 10 \mathrm{mM}\right.$ Tris pH 7.5, $2 \mathrm{mM} \mathrm{Mg}_{2} \mathrm{Cl}_{2}$ and $1 \%$ Triton $\mathrm{X}-100)$ for $30 \mathrm{~min}$ at $4^{\circ} \mathrm{C}$. Cell lysates were precleaned with normal mouse/rabbit IgG and $\mathrm{A} / \mathrm{G}$ beads (Santa Crus) for $2 \mathrm{~h}$ at $4^{\circ} \mathrm{C}$. Immunoprecipitation was performed using antibodies against Robo1, p85 and mouse/rabbit lgG overnight at $4^{\circ} \mathrm{C}$. The antigen-antibody complex was immobilized with $\mathrm{A} / \mathrm{G}$

beads for $2 \mathrm{~h}$ at $4^{\circ} \mathrm{C}$. After washed with wash buffer $\left(150 \mathrm{mM} \mathrm{NaCl}, 10 \mathrm{mM}\right.$ Tris pH 7.5, $2 \mathrm{mM} \mathrm{Mg}_{2} \mathrm{Cl}_{2}$ and $0.1 \%$ Triton $\mathrm{X}-100$ ), proteins were eluted by addition of loading dye and boiling at $95^{\circ} \mathrm{C}$ for $2 \mathrm{~min}$. The proteins were subjected to SDS-PAGE analysis followed by immunoblotting with various antibodies.

\section{Immunofluorescence}

Control and shRobo 1 of CNE1/IR cells were seeded on coverslips and treated with rSlit2. The cells were washed with PBS, fixed in $4 \%$ paraformaldehyde for $10 \mathrm{~min}$, washed with PBS and permeabilized in $0.2 \%$ Triton X-100 for 15 min. After washed with PBS, cells were blocked with PBS containing 10\% FBS for $2 \mathrm{~h}$ before incubation with the primary antibody as indicated anti- $\beta$-catenin overnight at $4^{\circ} \mathrm{C}$. The cells were incubated for $1 \mathrm{~h}$ with a fluorochrome-conjugated secondary antibody (Alexa Fluor 488 anti-mouse). Coverslips with stained cells were then washed with PBS, stained with 4',6-Diamidino-2-phenylindole (DAPI), and mounted onto glass slides with Vectashield mounting medium (Vector Laboratories, Burlingame, CA). For the primary tumor tissue sections, $100^{\circ} \mathrm{C}$ EDTA buffer was used for heat induced 
antigen retrieval. Slides were blocked in PBS containing 10\% FBS and then incubated with primary antibodies against $\beta$-catenin(Sigma) overnight at $4^{\circ} \mathrm{C}$. The slides were incubated for $1 \mathrm{~h}$ with fluorochrome-conjugated secondary antibody (Alexa Fluor 488 anti-mouse or Alexa Fluor 594 anti-rabbit). Slides were then stained with DAPI, and mounted onto glass slides with mounting medium.

\section{In vivo tumor metastasis experiment}

Male nude mice aged 4 weeks were obtained from the Laboratory Animal Center of the Fourth Military Medical University (Xi'an, china) and were maintained under specific pathogen-free conditions. For in vivo tumor metastasis assay, mice( $\mathrm{n}=5$ each group) were implanted with $1 \times 10^{6} / 100 \mu \mathrm{L}$ CNE1-IR/NC cells or $1 \times 10^{6} / 100 \mu \mathrm{L}$ CNE1-IR/shslit2 cells on day 0 by lateral tail vein injection. Five weeks after inject, Metastatic was quantified using a noninvasive bioluminescence In Vivo Imaging System (IVIS) (Xenogen) as described previously[11]. All mice were killed for necropsy and observed the lung metastasis via $\mathrm{H} \otimes \mathrm{E}$ assay.

\section{Statistical analysis}

All data were presented as means \pm standard deviation (SD). Differences between statistical comparisons were determined by Student's t-test and One-way ANOVA analysis via GraphPad Prism version 6.0. Asterisk (*)indicated statistical significance with $p$-value $\leq 0.05$. Asterisk $\left.{ }^{* *}\right)$ indicated statistical significance with $p$-value $\leq 0.01$.

\section{Results}

\section{Lethally irradiated NPC cells could promote migration and invasion of radioresistant NPC cell line CNE1/IR}

Firstly we performed colony formation assay to determine the opitimal dose used in this study. The result showed that the lethal irradiation dose (rate of survivaling cells<0.0001) for CNE1 cells was 16Gy

(Fig.1a). We further asked whether the lethally irradiated NPC cells could promote migration and invasive abilities of surviving NPC cells (CNE1-IR cells were considered as irradiated surviving cells) in the vicinity by changing the microenvironment. Migration and invasion assay showed that the migration (Fig.1b) and invasion abilities (Fig.1C) of CNE1-IR cells were significanly promoted $(P<0.01)$ when CNE1-IR was cocultured with lethally irradiated CNE1 cells.

\section{Slit2 was overexpressed in the supernatant oflethally irradiated NPC cell lines}

In order to examine whether the expression of Slit2 was altered in the supernatant of lethally irradiated CNE1 cells, we detected the expression of Slit2 in the lethally irradiated and unirridated control CNE1 cells. The expression of Slit2 in the supernatant of lethally irradiated CNE1 cells by ELSA assay confirmed that Slit2 in the supernatant of lethally irradiated CNE1 cells was significantly higher than control cells (Fig.2a). qRT-PCR analysis also showed that the expressions of Slit2 was significantly overexpressed in 
irradiated NPC cells compared with unirridated control CNE1 cells (Fig.2b). Western blot analysis also confirmed the significantly overexpression of Slit2 protein level (Fig.2c\&d).

\section{Slit2 could promote migration and invasion abilities of NPC cell lines}

Two stable cell lines of CNE1-IR infected with Slit2 shRNA lentivirus (LV3-shSlit2) or control shRNA lentivirus (LV3-NC) were constructed. qRT-PCR and Western blot confirmed the downregulation of Slit2 expression in CNE1-IR/ shSlit2 cells (Fig.3a\&b). rSlit2 were used to upregulate the expression of Slit2. Subsequently, we further examined the role of Slit2 in promoting metastasis of NPC cell lines. Migration assay indicated that the migration abilities of CNE1-IR cells treated with rSlit2 was significantly increased compared with control cells. In contrast, migration abilities of the CNE1-IR/shSlit2 was significantly decreased in comparison to control cells (Fig.3c). Likewise, invasion assay also showed the rSlit2 could enhance the invasive ability of CNE1-IR cells, whereas down-regulated Slit2 could inhibit CNE1-IR cells invasion (Fig.3d).

\section{Slit2 could promote NPC metastasis via Robo1 receptor}

We next examined which Robo receptors were involved in Slit2 mediated NPC metastasis. Four Robo receptors(Robo1-4) have been identified in mammals on the basis of structural similarities. Although it was once thought that all Robo receptors could bind to Slits, mammalian Robo3 and Robo4 lack the key amino acid residues required for Slit binding[12]. qRT-PCR and Western blot analysis indicated that the expressions of both Robo1 and Robo2 could be detected in CNE1 and CNE1-IR cell lines (Fig.4a). Two stable CNE1-IR cells with Robo1 and Robo2 depleted using a lentivirus shRNA system have been established, which was confirmed by qRT-PCR and Western blot analysis(Fig.4b\&c). Subsequently, Migration analysis showed that addition of rSlit2 could enhance cell migratioin ability. In contrast, Robo1depleted cells showed decreased migration and did not respond to rSlit2 (Fig.4d). When rSlit2 was added to CNE1-IR/shRobo2 cells, the numbers of transmembrance CNE-IR cells increased by about $26 \%$ compared with CNE1-IR/NC cells (Fig.4f). Invasion assay showed the similar results consistent with migration analysis (Fig.4e\&g). Taken together, these results demonstrated that Slit2 secreted from lethally irradiated NPC cells could enhance NPC cell lines migration and invasion via Robo1 receptor.

\section{Slit2/Robo1 signaling enhances nuclear translocation of $\beta$-catenin via PI3K/Akt pathway}

Slit2/Robo1 signaling has been associated with Akt phosphorylation and upregulation of $\beta$-catenin activity. Migration and invasion analysis showed that the migration and invasive abilites of CNE1-IR cells could be decreased by PI3K inhibitor (Wortmannin, $5 \mathrm{nM}$ ). Meanwhile rSlit2 induced increase of migration and invasion abilities could be attenuated by Wortmannin (Fig.5a\&b). Co-immune precipitation (Co-IP) assay with anti-Robo1 antibody indicated that Robo1 was immunoprecipitated with p85, a subunit of $\mathrm{PI} 3 \mathrm{~K}$, indicating the interaction of Robo1 and p85 (Fig. 5c). The phospho-Akt activity was increased in rSlit2 treated cells, but the effect was attenuated in Robo1-depleted cells and total expression of AKT and $\beta$-catenin was not significant changed (Fig.5d). Consistently, using cellular fractionation and immunoblotting, we found that $\beta$-catenin expression was increased in rSlit2 treated CNE1-IR cells, but the 
effect was attenuated in Robo1-depleted cells in cell nucleus, not in cytoplasm (Fig.5e) . Likewise, upon rSlit2 treatment, an enhanced nuclear localization of $\beta$-catenin was detected by immunofluorescence assay, but the effect was attenuated in Robo1-depleted cells (Fig.5f). Taken together, these results suggested that activation of Robo1 by Slit2 from lethally irradiated NPC cells could enhance NPC metastasis via facilitating $\mathrm{PI} 3 \mathrm{~K} / \mathrm{AKT} / \beta$-catenin pathway

\section{Slit2 promotes NPC metastasis in vivo}

To investigate whether Slit2 was able to promote NPC cell lines metastasis in vivo, we established luciferase-expressing CNE1-IR/NC-luc and CNE1-IR/shSlit2-Luc cell lines. Cells were intravenously injected into SCID mice ( $\mathrm{n}=5$ per group), and tumor metastasis was photographed by bioluminescence imaging. As shown in Fig.6a, knockdown of Slit2 significantly suppressed lung and liver metastasis. Mice were sacrificed 5 weeks post-injection, and the results showed that the number of lung and liver metastatic nodules (Fig. 6b\&c) generated by CNE1-IR/shSlit2-Luc cells was significantly fewer than that of CNE1-IR/NC-Luc cells. H\&E staining also revealed that knockdown of Slit2 significantly suppressed lung and liver metastasis (Fig.6d\&e). These results indicateed that Slit2 knockdown could suppress lung and liver metastasis in vivo

\section{Discussion}

Radiation therapy is the main treatment modality for nasopharyngeal carcinoma. However, distant metastases may occur partially due to the enhanced invasiveness properties of the surviving cancer cells resistant to radiotherapy. In vivo and in vitro studies have indicated that irradiation could promote the invasion of the surviving cancer cells after irradiation[7], suggesting alteration of tumor microenvironment may play an important role in this process [13]. We for the first time constructed a coculture cell model and found that the supernatant from the lethally irradiated NPC CNE1 cell line could promote migration and invasion ability of radioresistant NPC cell line CNE1-IR. We further provided evidence that slit2, a secrected factor released from irradiated dying cancer cells could promote metastasis of resistant NPC cells through PI3K/AKT/ $\beta$-catenin pathway. We conclude that approaches targeting tumor microenvironment in combination with conventional cancer therapy could inhibit tumor metastasis.

Several studies have demonstrated that ionizing radiation might promote metastastic abilities of surviving cancer cells[7]. Radiation-enhanced cell invasion was shown to be associated to EMT[14]. Our previous studies also indicated that irridated cancer cells presented the mesenchymal phenotype, and the migratory and invasive capabilities were strengthened. Also, EMT induced by irradiation could be reversed through inhibition of TGF- $\beta$ signaling[8]. Another study indicated that CMET may be implied in radiationenhanced cell invasion of pancreatic tumor cells when their stromal microenvironment was irradiated[5]. These studies indicated that the irradiated microenvironment might contribute to the irradiation-enhanced cell invasion. The microenvironment could be influenced by the irridated dying cancer cell. Huang et al showed that the irridated dying cancer cells could promote the repopulation of cancer cells undergoing 
radiotherapy and caspase 3 was involved in this process. Yu et al found Sox 2 overexpression enhanced the growth-stimulating effect of irradiated dying pancreatic cancer cells on living cancer cells[15]. Another study have consistent results showing dying caner cells promote proliferation of living cancer cells in pancreatic ductal adenocarcinoma[16]. In this study, we found that the supernatant from lethally irradiated dying NPC cell lines could promote migration and invasion ability of radioresistant NPC cell lines, which is consistent with previous studies. Taken together, these results confirmed the the existence of a cell death-induced tumor repopulation or metastasis, and further provide the evidence that alteration of tumor microenvironment plays a key role in tumor malignant biological behaviors. Tumor microenvironment altered by irradiated dying cancer cells may be a promising target to reduce cancer metastasis.

We further provided evidence that Slit2, a secrected factor released from irradiated dying cancer cells could promote metastasis of NPC cells through PI3K/AKT/ $\beta$-catenin pathway. Slit2 is a large secreted protein with multi-functions during neural development and elongation[17, 18]. The roles of Slit2 in cancer have been extensively studied[19-22]. Our previous study suggested Slit2 enriched by the absence of miR-218 could promote gastric cancer invasion [10]. Here, We found that knockdown of Slit2 could inhibit NPC cell growth and metastasis in vitro and in vivo, while adding rSlit or the supernatant of lethally irridated CNE1 cells could promote cell growth and metastasi. Robo1 and Robo2 are two receptors of the secretory protein Slit2. It was previously reported that Slit2 and Robo1 are expressed in a large number of malignant solid tumors and the binding of the functional region of Slit2 to Robo1 could initiate a cascade of events leading to altered cell proliferation, migration, apoptosis, and angiogenesis[12, 23, 24]. Up-regulation of Robo1 has been reported to be correlated with poor prognosis of nasopharyngeal cancer (NPC)[20, 23]. Our study indicated that both Robo1 and Robo2 play a role in NPC metastasis and Slit2 secreted from lethally irradiated NPC cells could enhance NPC cell lines migration and invasion via Robo1 receptor.

Slit2/Robo1 signaling has been associated with Akt phosphorylation and upregulation of $\beta$-catenin activity[25, 26]. Yuan et al found Slit2/Robo1 promotes tumor invasion partly by upregulation of MMP2 after activation of PI3K/Akt signaling pathway in human hepatocellular carcinoma cell line[25]. PI3K/Akt/ $\beta$-catenin signaling is highly activated in NPC [27] Activation of the PI3K/Akt signaling pathway results in aberrant cell proliferation, tumorigenesis and metastatic competence[28-30]. Meng et al found that down-regulation of MACC1 inhibits proliferation and tumourigenicity of NPC cell lines through Akt/ $\beta$ catenin pathway[31]. One study showed that UBE2T promotes metastasis of NPC cell lines by activating the AKT/GSK3 $\beta / \beta$-catenin pathway[32]. Consistent with these reports, our study showed that activation of Slit2/Robo1 promotes Akt phosphorylation and enhances $\beta$-catenin translocation from cytoplasm to nucleus. Furthermore, we also demonstrated that $\beta$-catenin accumulates in membrane instead of nucleus in NPC cell with higher Robo1 and Slit2 expression, supporting the importance of the Slit2/Robo1mediated activation of Akt/ $\beta$-catenin activity in NPC metastasis.

\section{Conclusion}


In conclusion, our data showed that lethally irradiated NPC cells could promote migration and invasion of radioresistant NPC cell lines. We further provided evidence that Slit2, a secreted factor released from irradiated dying NPC cells could promote metastasis of living/resistent NPC cells through PI3K/AKT/ $\beta$ catenin pathway. Tumor microenvironment altered by irradiated dying cancer cells may be promising target to treat cancer metastasis.

\section{Abbreviations}

NPC

Nasopharyngeal carcinoma;

ELISA

Enzyme-linked immune- sorbent assay;

Co-IP

Co-immunoprecipitation;

shRNA

short hairpin RNA;

PI3K

phosphatidy linositol 3-kinase;

SDS-PAGE

sodium dodecyl sulfate-polyacrylamide gelelectroph oresis;

CCRT

concurrent chemoradiation;

PGE2

prostaglandin E2;

PBS

phosphate buffered saline;

NC

Negative control;

EMT

epithelial-mesenchymal transition;

TGF- $\beta$

transforming growth factor-beta;

MMP2

matrix metalloproteinase 2;

UBE2T

ubiquitin conjugating enzyme E2T;

GSK3 $\beta$

glycogen synthase kinase 3 beta.

\section{Declarations}




\section{Ethics approval and consent to participate}

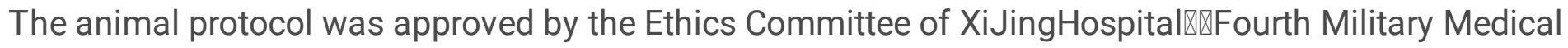
University $\mathbb{X}$ i'an, China).

\section{Consent for publication}

Not applicable.

\section{Availability of data and materials}

The datasets used and/or analyzed during the current study are available from the corresponding author on reasonable request

\section{Competing interests}

The authors declare that there is no conflict of interest

\section{Funding}

This work was supported by the National Natural Science Foundation of China (Grant No. 81372421 . Grant No. 81872699 and 81802977)

\section{Acknowledgements}

Not applicable

\section{Author contributions}

RM, LZ and MS conceived and designed the experiments; RM and HY wrote the manuscript; RM performed the experiment; $\mathrm{RM}$ and $\mathrm{JH}$ analyzed the data, $\mathrm{LZ}$ and $\mathrm{CH}-\mathrm{L}$ revised the paper.

\section{Contributor Information}

Mei Shi, E-mail囚mshi82@hotmail.com

Li-Na Zhao, E-mail囚zhaolina_zIn@163.com

Rui Ma, E-mail囚marui0913@163.com

Hua Yang, E-mail囚yanghuafmmu@163.com

Chang-Hao Liu, E-mail『cliu@fmmu.edu.cn

Jing Hu, E-mail囚hujing1999@163.com 


\section{References}

1. Al-Sarraf M, LeBlanc M, Giri PG, Fu KK, Cooper J, Vuong T, Forastiere AA, Adams G, Sakr WA, Schuller $D E$, et al. Chemoradiotherapy versus radiotherapy in patients with advanced nasopharyngeal cancer: phase III randomized Intergroup study 0099. Journal of clinical oncology: official journal of the American Society of Clinical Oncology. 1998;16(4):1310-7.

2. Zhao LN, Zhou B, Shi M, Wang JH, Xiao F, Xu M, Luo SQ, Xue Y, Li JP, Tan LN. Clinical outcome for nasopharyngeal carcinoma with predominantly WHO II histology treated with intensity-modulated radiation therapy in non-endemic region of China. Oral Oncol. 2012;48(9):864-9.

3. Wang J, Shi M, Hsia Y, Luo S, Zhao L, Xu M, Xiao F, Fu X, Li J, Zhou B, et al. Failure patterns and survival in patients with nasopharyngeal carcinoma treated with intensity modulated radiation in Northwest China: a pilot study. Radiation oncology (London England). 2012;7:2.

4. Wee CW, Keam B, Heo DS, Sung MW, Won TB, Wu HG. Locoregionally advanced nasopharyngeal carcinoma treated with intensity-modulated radiotherapy plus concurrent weekly cisplatin with or without neoadjuvant chemotherapy. Radiation oncology journal. 2015;33(2):98-108.

5. Ohuchida K, Mizumoto K, Murakami M, Qian LW, Sato N, Nagai E, Matsumoto K, Nakamura T, Tanaka M. Radiation to stromal fibroblasts increases invasiveness of pancreatic cancer cells through tumorstromal interactions. Cancer research. 2004;64(9):3215-22.

6. Milas L, Hunter N, Peters LJ. The tumor bed effect: dependence of tumor take, growth rate, and metastasis on the time interval between irradiation and tumor cell transplantation. Int $\mathrm{J}$ Radiat Oncol Biol Phys. 1987;13(3):379-83.

7. Fujita M, Yamada S, Imai T. Irradiation induces diverse changes in invasive potential in cancer cell lines. Sem Cancer Biol. 2015;35:45-52.

8. Zhou YC, Liu JY, Li J, Zhang J, Xu YQ, Zhang HW, Qiu LB, Ding GR, Su XM, Mei S, et al. lonizing radiation promotes migration and invasion of cancer cells through transforming growth factor-betamediated epithelial-mesenchymal transition. Int J Radiat Oncol Biol Phys. 2011;81(5):1530-7.

9. Huang Q, Li F, Liu X, Li W, Shi W, Liu FF, O'Sullivan B, He Z, Peng Y, Tan AC, et al. Caspase 3-mediated stimulation of tumor cell repopulation during cancer radiotherapy. Nature medicine. 2011;17(7):8606.

10. Tie J, Pan Y, Zhao L, Wu K, Liu J, Sun S, Guo X, Wang B, Gang Y, Zhang Y, et al. MiR-218 inhibits invasion and metastasis of gastric cancer by targeting the Robo1 receptor. PLoS Genet. 2010;6(3):e1000879.

11. Ho MY, Liang SM, Hung SW, Liang CM. MIG-7 controls COX-2/PGE2-mediated lung cancer metastasis. Cancer research. 2013;73(1):439-49.

12. Rama N, Dubrac A, Mathivet T, Ni Charthaigh RA, Genet G, Cristofaro B, Pibouin-Fragner L, Ma L, Eichmann A, Chedotal A. Slit2 signaling through Robo1 and Robo2 is required for retinal neovascularization. Nature medicine. 2015;21(5):483-91. 
13. Oudin MJ, Weaver VM. Physical and Chemical Gradients in the Tumor Microenvironment Regulate Tumor Cell Invasion, Migration, and Metastasis. Cold Spring Harb Symp Quant Biol. 2016;81:189205.

14. Fujita M, Otsuka Y, Yamada S, Iwakawa M, Imai T. X-ray irradiation and Rho-kinase inhibitor additively induce invasiveness of the cells of the pancreatic cancer line, MIAPaCa-2, which exhibits mesenchymal and amoeboid motility. Cancer Sci. 2011;102(4):792-8.

15. Yu Y, Tian L, Feng X, Cheng J, Gong Y, Liu X, Zhang Z, Yang X, He S, Li CY, et al. elF4Ephosphorylation-mediated Sox2 upregulation promotes pancreatic tumor cell repopulation after irradiation. Cancer letters. 2016;375(1):31-8.

16. Cheng J, Tian L, Ma J, Gong Y, Zhang Z, Chen Z, Xu B, Xiong H, Li C, Huang Q. Dying tumor cells stimulate proliferation of living tumor cells via caspase-dependent protein kinase Cdelta activation in pancreatic ductal adenocarcinoma. Molecular oncology. 2015;9(1):105-14.

17. Liu X, Lu Y, Zhang Y, Li Y, Zhou J, Yuan Y, Gao X, Su Z, He C. Slit2 regulates the dispersal of oligodendrocyte precursor cells via Fyn/RhoA signaling. J Biol Chem. 2012;287(21):17503-16.

18. Wang G, Li Y, Wang XY, Han Z, Chuai M, Wang LJ, Ho Lee KK, Geng JG, Yang X. Slit/Robo1 signaling regulates neural tube development by balancing neuroepithelial cell proliferation and differentiation. Experimental cell research. 2013;319(8):1083-93.

19. Feng Y, Feng L, Yu D, Zou J, Huang Z. srGAP1 mediates the migration inhibition effect of Slit2-Robo1 in colorectal cancer. Journal of experimental clinical cancer research: CR. 2016;35(1):191.

20. Li J, Zhou C, Wang G, Wang S, Ni S, Ye M, Zhang J. Promoter hypermethylation of SLIT2 is a risk factor and potential diagnostic biomarker for nasopharyngeal carcinoma. Gene. 2018;644:74-9.

21. Yang Y, Ding L, Hu Q, Xia J, Sun J, Wang X, Xiong H, Gurbani D, Li L, Liu Y, et al. MicroRNA-218 functions as a tumor suppressor in lung cancer by targeting IL-6/STAT3 and negatively correlates with poor prognosis. Mol Cancer. 2017;16(1):141.

22. Zhao SJ, Shen YF, Li Q, He YJ, Zhang YK, Hu LP, Jiang YQ, Xu NW, Wang YJ, Li J, et al. SLIT2/ROBO1 axis contributes to the Warburg effect in osteosarcoma through activation of SRC/ERK/CMYC/PFKFB2 pathway. Cell death disease. 2018;9(3):390.

23. Alajez NM, Lenarduzzi M, Ito E, Hui AB, Shi W, Bruce J, Yue S, Huang SH, Xu W, Waldron J, et al. MiR218 suppresses nasopharyngeal cancer progression through downregulation of survivin and the SLIT2-ROB01 pathway. Cancer research. 2011;71(6):2381-91.

24. Maiti GP, Ghosh A, Mondal P, Ghosh S, Chakraborty J, Roy A, Roychowdhury S, Panda CK. Frequent inactivation of SLIT2 and ROBO1 signaling in head and neck lesions: clinical and prognostic implications. Oral surgery, oral medicine, oral pathology and oral radiology 2015, 119(2):202-212.

25. Yuan M, Guo H, Li J, Sui C, Qin Y, Wang J, Khan YH, Ye L, Xie F, Wang H, et al. Slit2 and Robo1 induce opposing effects on metastasis of hepatocellular carcinoma Sk-hep-1 cells. Int $\mathrm{J}$ Oncol. 2016;49(1):305-15.

26. Tseng RC, Lee SH, Hsu HS, Chen BH, Tsai WC, Tzao C, Wang YC. SLIT2 attenuation during lung cancer progression deregulates beta-catenin and E-cadherin and associates with poor prognosis. 
Cancer research. 2010;70(2):543-51.

27. Zhang G, Wang W, Yao C, Zhang S, Liang L, Han M, Ren J, Qi X, Zhang X, Wang S, et al. Radiationresistant cancer stem-like cell properties are regulated by PTEN through the activity of nuclear betacatenin in nasopharyngeal carcinoma. Oncotarget. 2017;8(43):74661-72.

28. Gluck S. Consequences of the Convergence of Multiple Alternate Pathways on the Estrogen Receptor in the Treatment of Metastatic Breast Cancer. Clin Breast Cancer. 2017;17(2):79-90.

29. Tan M, Xu J, Siddiqui J, Feng F, Sun Y. Depletion of SAG/RBX2 E3 ubiquitin ligase suppresses prostate tumorigenesis via inactivation of the PI3K/AKT/mTOR axis. Mol Cancer. 2016;15(1):81.

30. Xu G, Dang C. CMTM5 is downregulated and suppresses tumour growth in hepatocellular carcinoma through regulating PI3K-AKT signalling. Cancer cell international. 2017;17:113.

31. Meng F, Li H, Shi H, Yang Q, Zhang F, Yang Y, Kang L, Zhen T, Dai S, Dong Y, et al. MACC1 downregulation inhibits proliferation and tumourigenicity of nasopharyngeal carcinoma cells through Akt/beta-catenin signaling pathway. PloS one. 2013;8(4):e60821.

32. Hu W, Xiao L, Cao C, Hua S, Wu D. UBE2T promotes nasopharyngeal carcinoma cell proliferation, invasion, and metastasis by activating the AKT/GSK3beta/beta-catenin pathway. Oncotarget. 2016;7(12):15161-72.

Figures

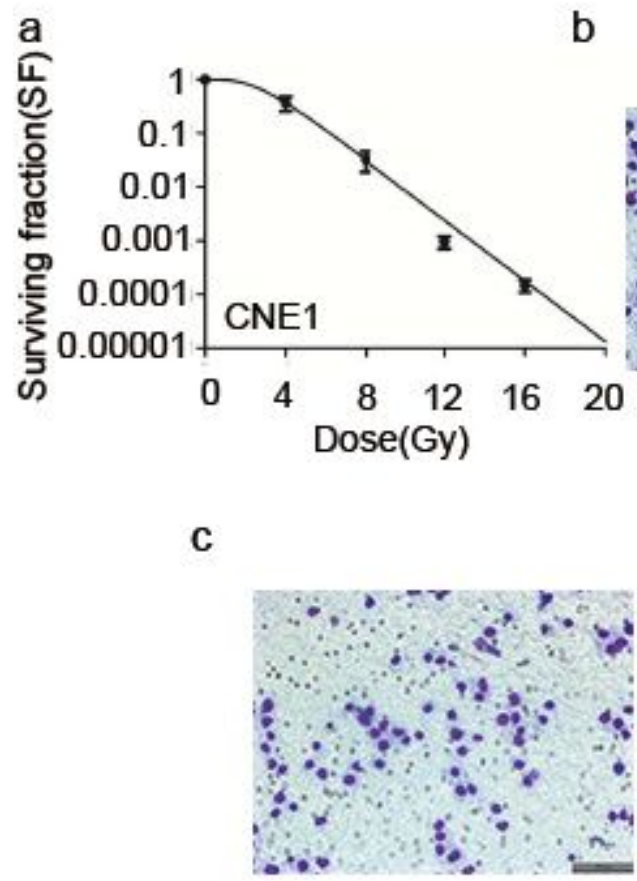

CNE1-IR/Control

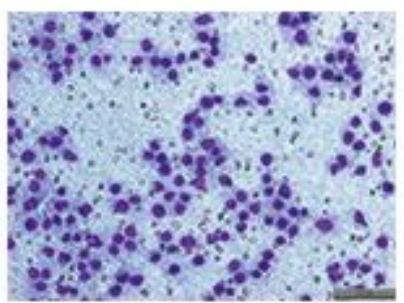

CNE1-IR/Lethal-CNE1

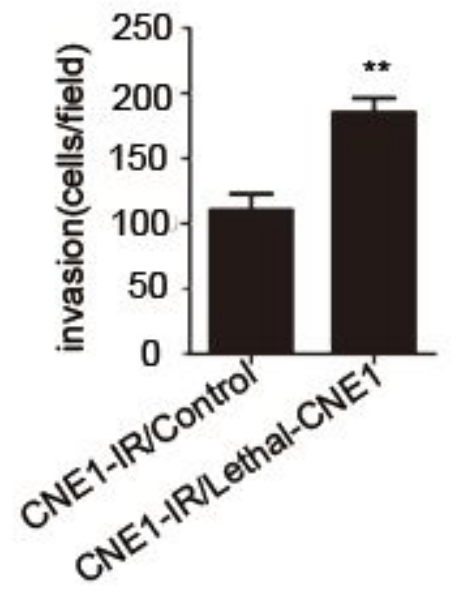


Figure 1

Lethally irradiated NPC cells could promote CNE1-IR cell migration and invasion. a: Clone formation assay using CNE1 cells to confirm a lethal dose of ionizing radiation; b: Migration assay using CNE1-IR co-cultured with Lethal CNE1 cells; c:Invasion assay using CNE1-IR co-cultured with Lethal CNE1 cells. *, $\mathrm{P}<0.05 ; * *, \mathrm{P}<0.01$.

a

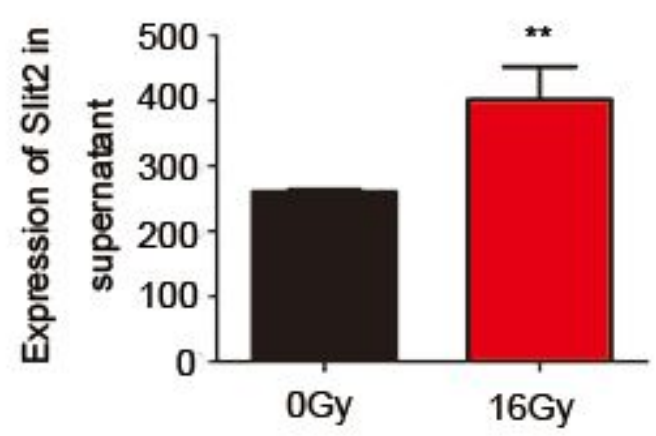

C

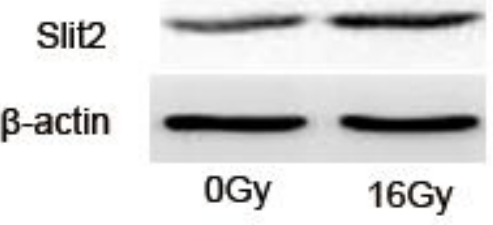

b

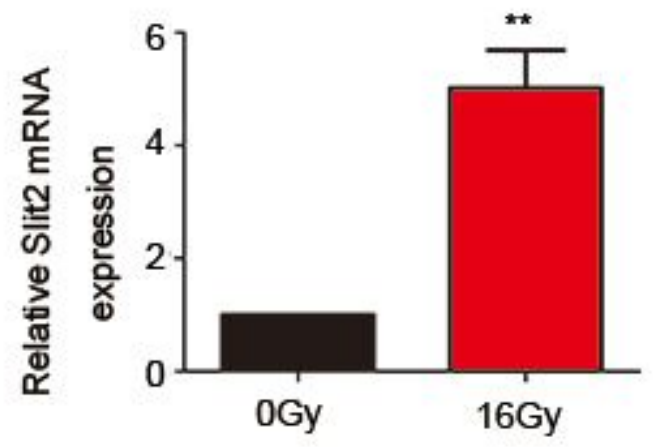

d

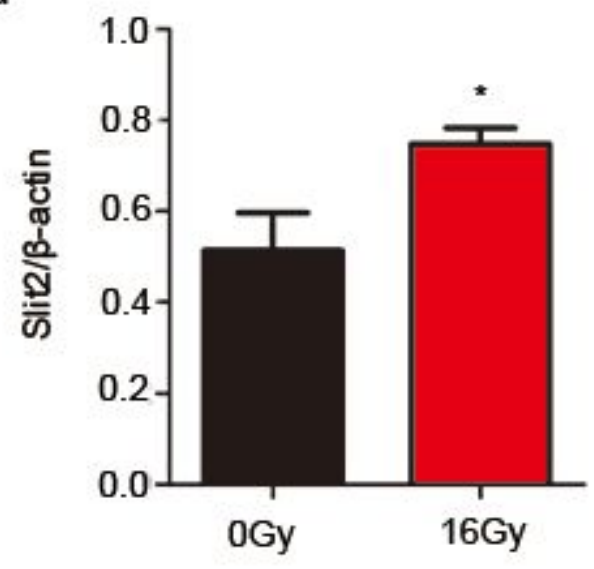

\section{Figure 2}

Slit2 expression in lethally irradiated and control CNE1 cell lines. a: ELISA assay analysis of Slit2 expression in the supernatant. b: Slit2 mRNA expression by qRT-PCR analysis; $c$ and d:Western blot analysis of Slit2 expression in protein lysates. ${ }^{*} \mathrm{P}<0.05 ; * * \mathrm{P}<0.01$. 

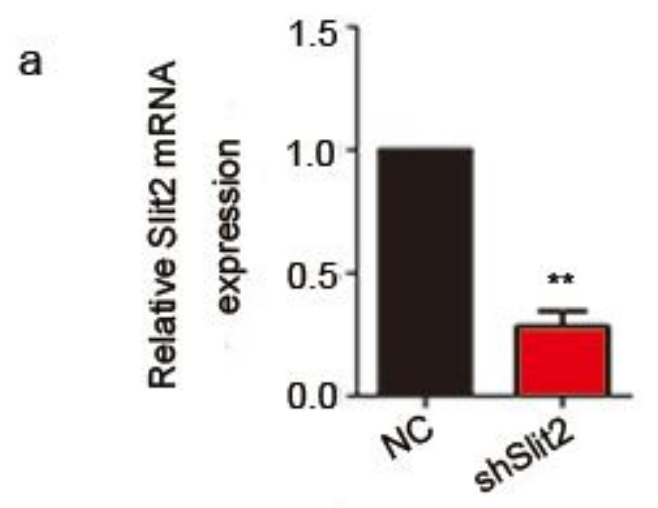

b

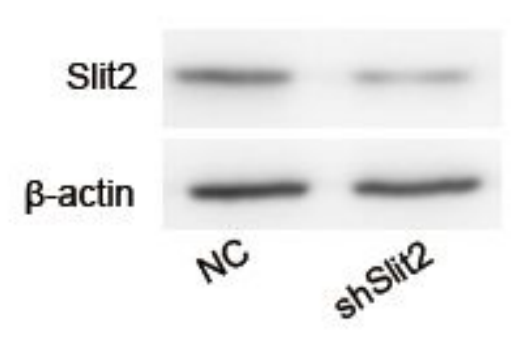

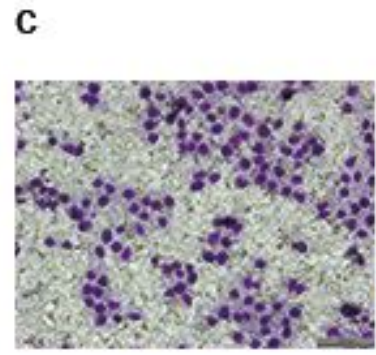

NC

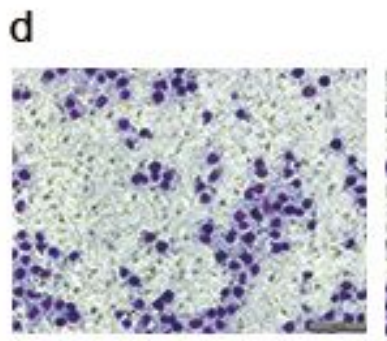

NC

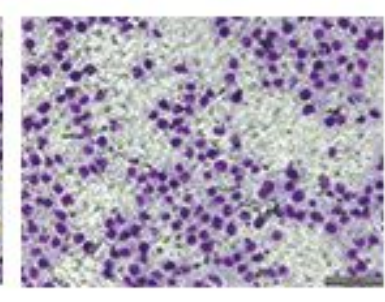

rSlit2

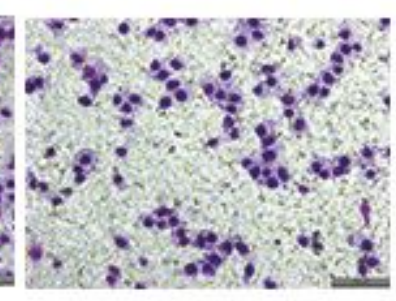

shSlit2
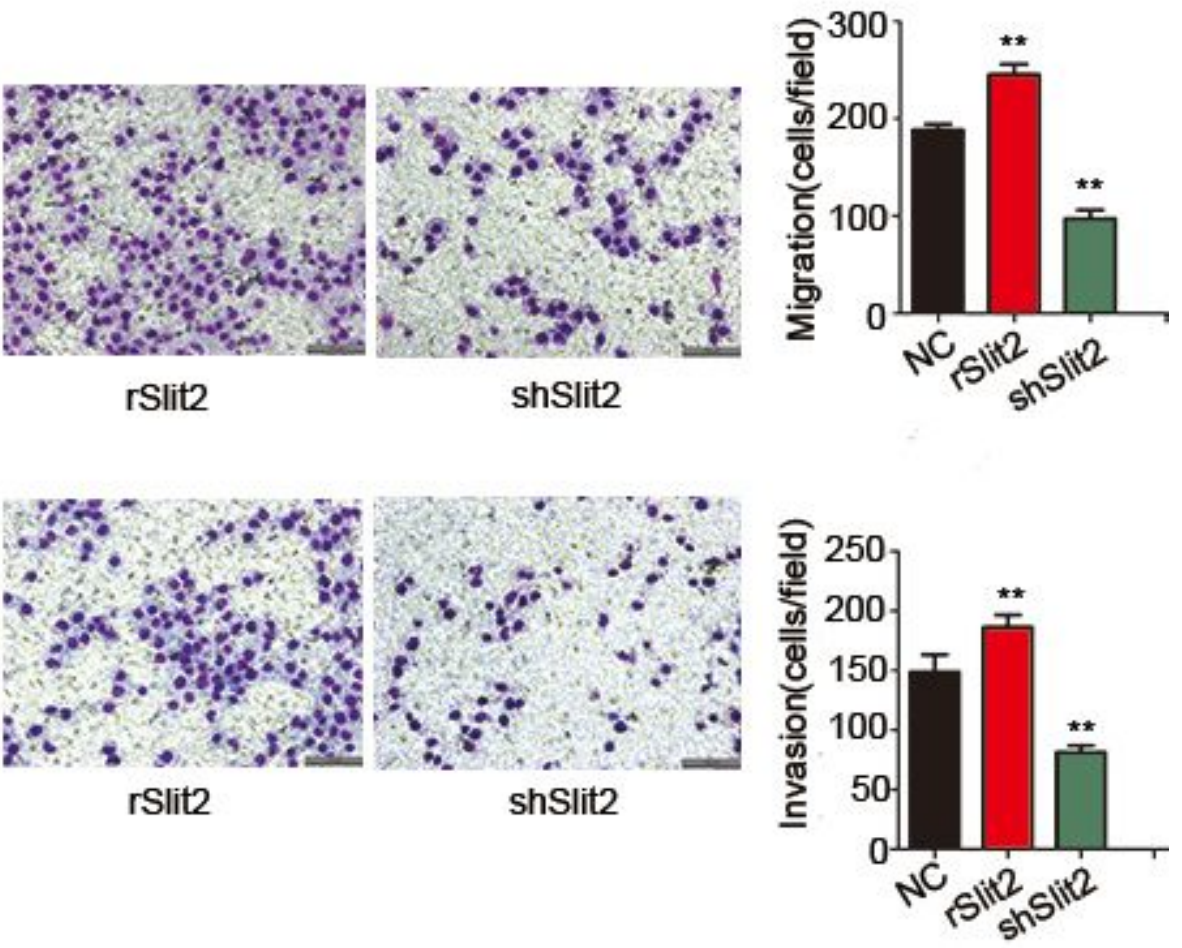

Figure 3

Slit2 could promote migration and invision abilities of NPC a and b: Down-regulation of Slit2 expression infected with LV3-NC and LV3-shSlit2 in CNE1-IR cell lines were confirmed by Western blot analysis and qRT-PCR; c: Migration assay analysis of the migration abilities of CNE1-IR/shSlit2 cells and CNE1-IR cells treated rSlit2 $(50 \mathrm{ng} / \mathrm{ml})$; d:Invasion assay analysis of the invasive abilities of CNE1-IR/shSlit2 cells and CNE1-IR cells treated with rSlit2 $(50 \mathrm{ng} / \mathrm{ml}) *, P<0.05 ; * \star, P<0.01$. 
a

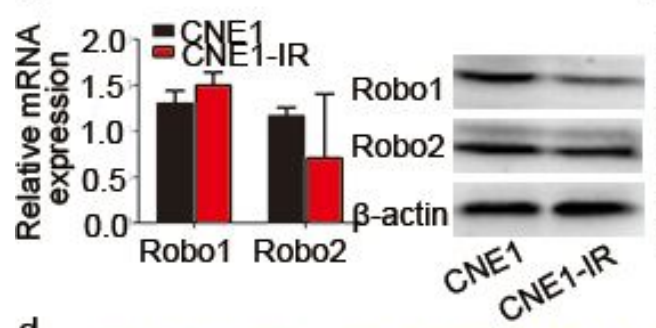

d

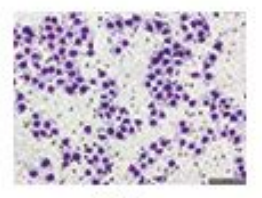

NC
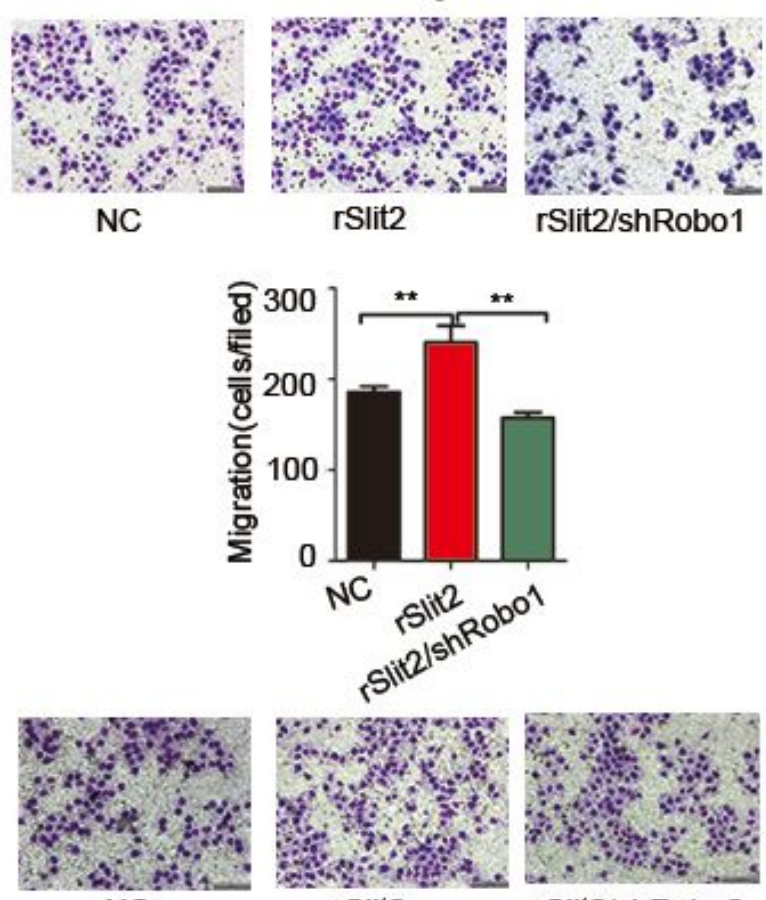

NC b

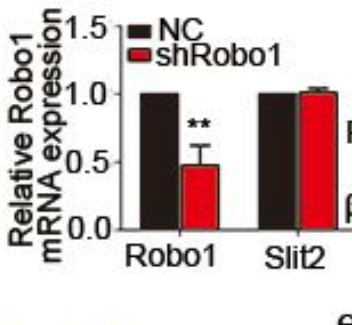

C

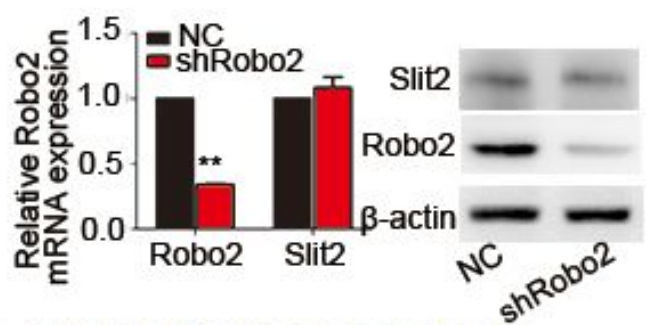

e
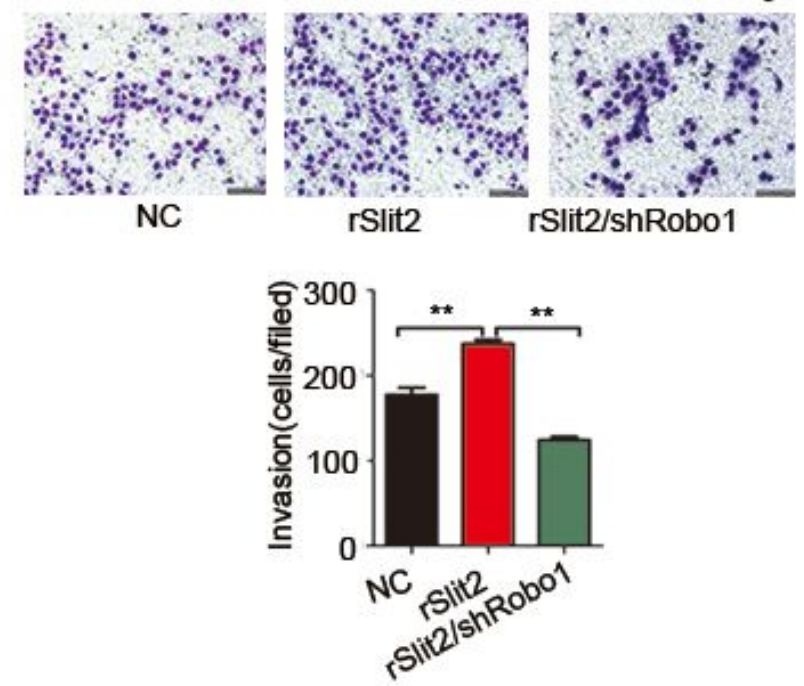

g
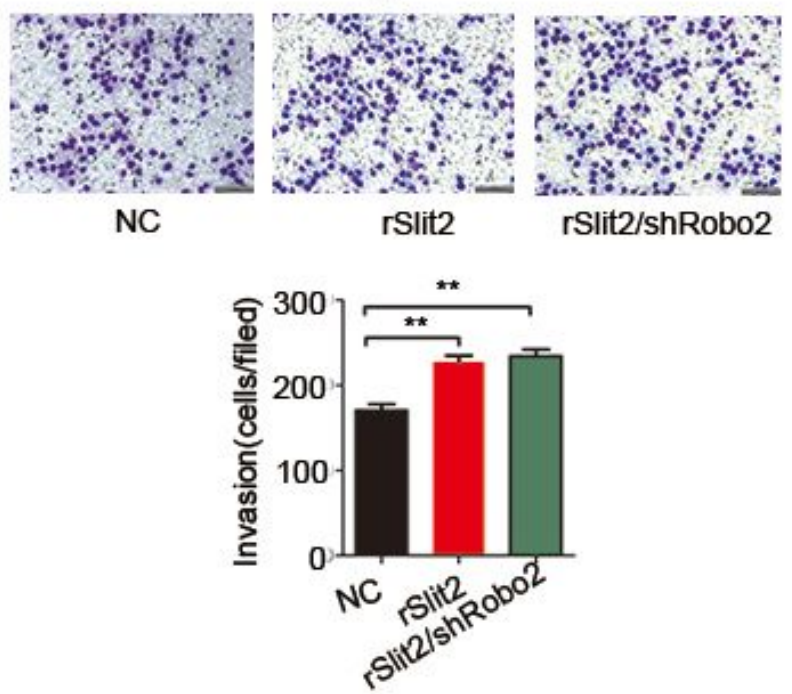

\section{Figure 4}

Slit2 enhance NPC cell lines metastasis via receptor Robo1 a. Robo1 and Robo2 expression in NPC cell lines CNE1 and CNE1-IR were examined by western blot and qRT-PCR;b and c qRT-PCR and Western blot analysis of Slit2, Robo expression in CNE1-IR cells infected with LV3-NC, LV3-shRobo1 and LV3-shRobo2; $d$ and $f$. Migration assay analysis of CNE1-IR cells infected with LV3-NC, LV3-shRobo1 and LV3-shRobo2 or addition of rSlit (50ng/ml);e and $\mathrm{g}$. lavasion assay analysis of CNE1-IR cells infected with LV3-NC, LV3shRobo1 and LV3-shRobo2 or addition of rSlit $(50 \mathrm{ng} / \mathrm{ml})$. These experiments were repeated three times,

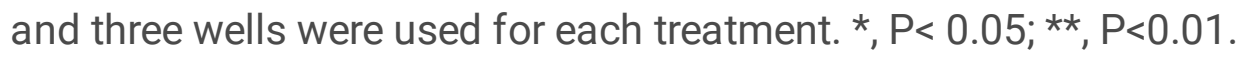


a
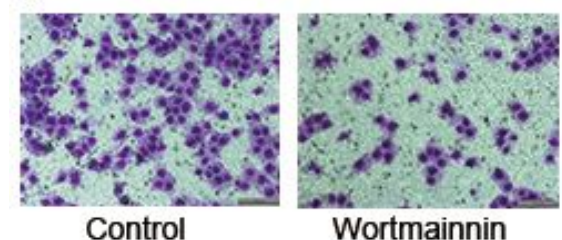

Wortmainnin
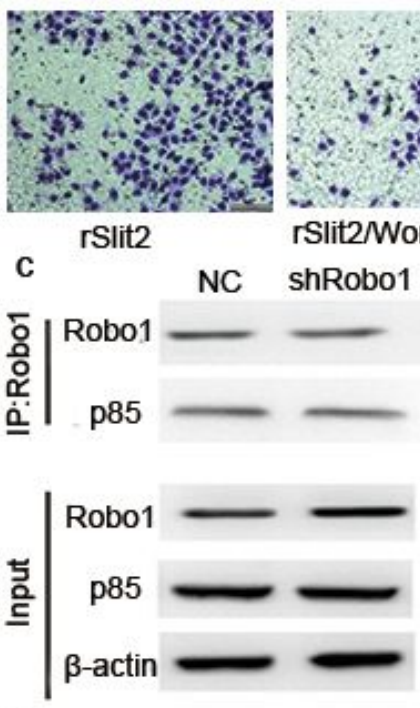

$\mathrm{e}$

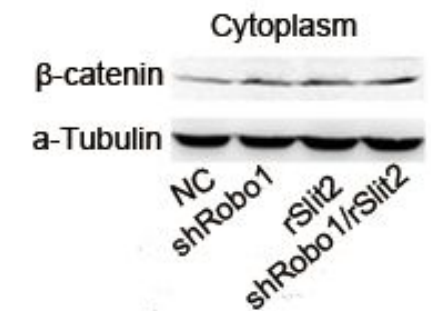

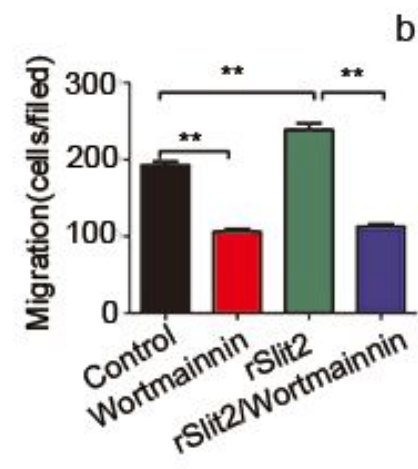

b

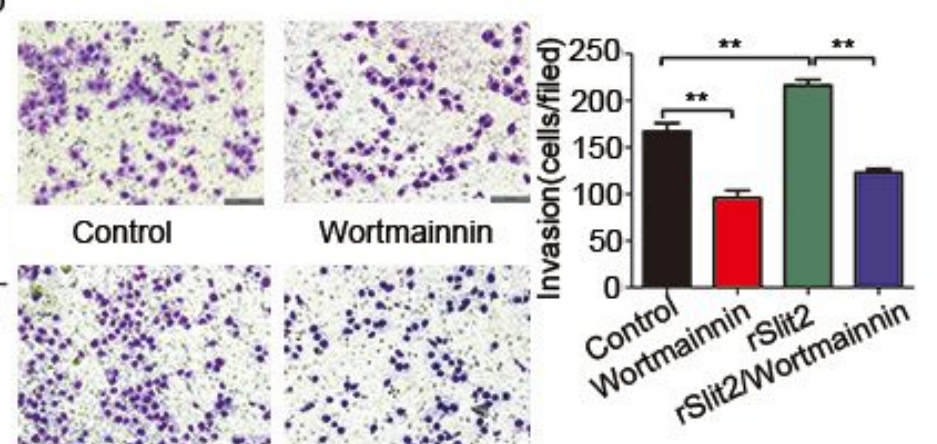

rSlit2

rSlit2/Wortmainnin
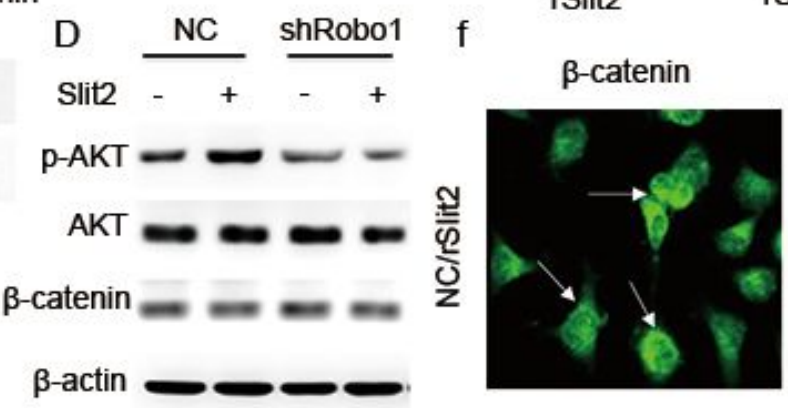

DAPI
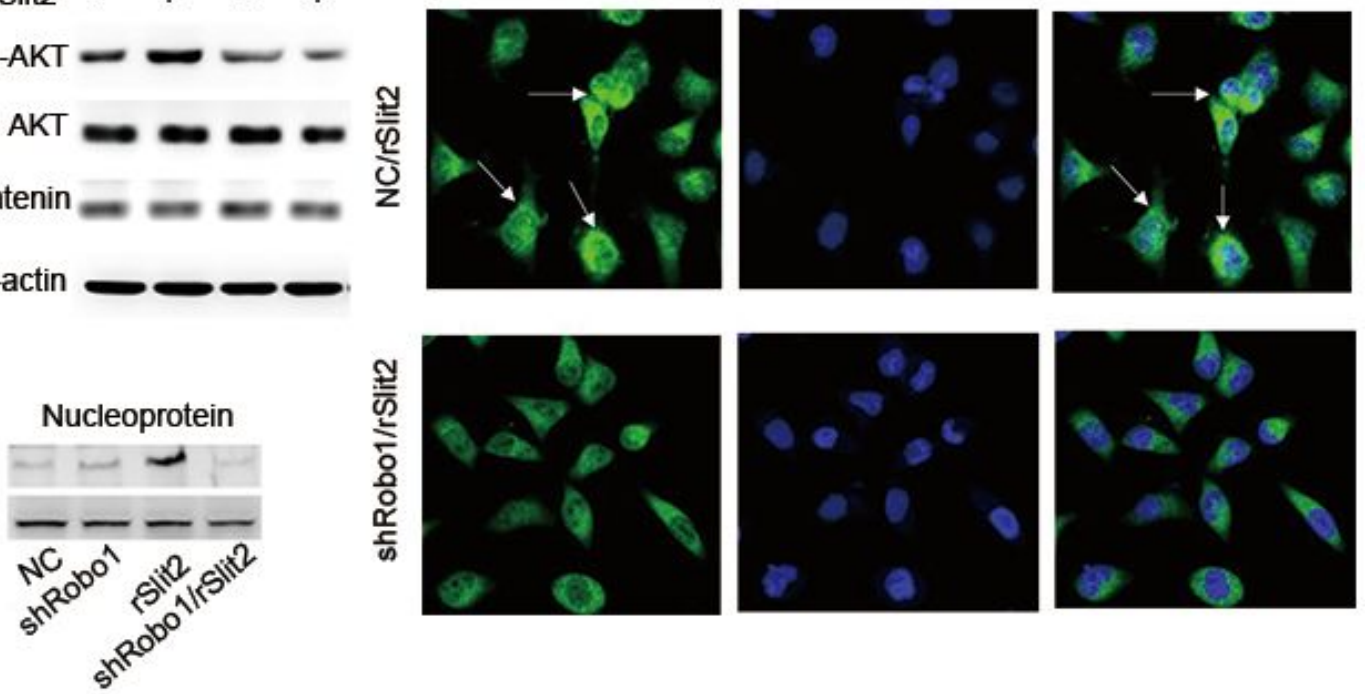

Figure 5

Slit2/Robo1 signaling enhances $\beta$-catenin translocation through PI3K/Akt pathway a and b: Migration and invasion assay analysis of the migration of CNE1-IR cells were decreased by PI3K inhibitor (Wortmannin 5nM); c: Co-immunoprecipitation (Co-IP) of Robo1 and p85 was detected using immunoblotting analysis. Normal IgG (IgG) was used as a negative Co-IP control.; d: Western blot analysis of phospho-Akt,Total AKT and $\beta$-catenin in NC and shRobo1CNE1-IR cells treated with rSlit2(50ng/ml). $\beta$-actin served as a loading control; e: Western blot assay of $\beta$-catenin in cytosolic and nuclear fractions of the NC and shRobo1 CNE1-IR cells. a-tubulin served as a loading control; $f$ : Immunofluorescence staining with antibody against $\beta$-catenin for NC and shRobo1 CNE1-IR cells after treated with rSlit2 (50ng/ml). *, $\mathrm{P}<0.05 ;$ **, $\mathrm{P}<0.01$. 
a

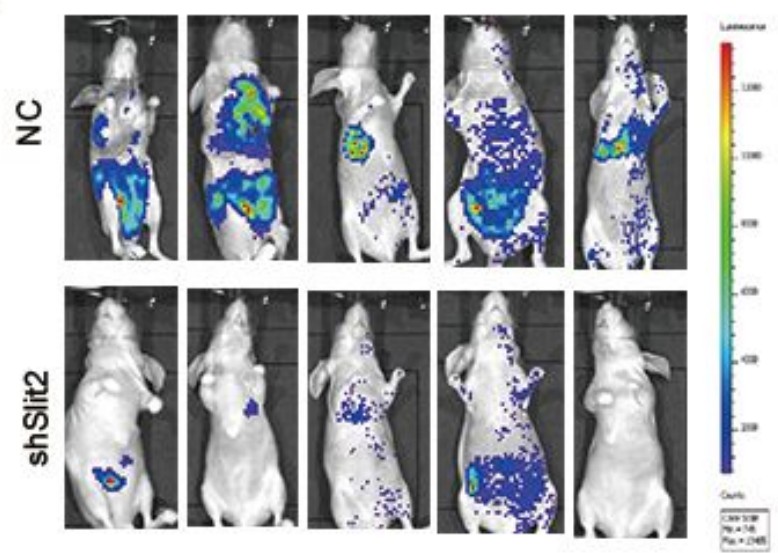

Lung

d

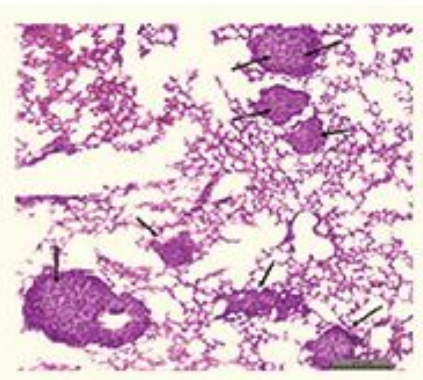

NC

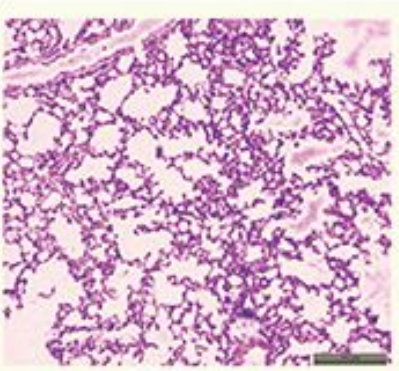

shSlit2

b

C
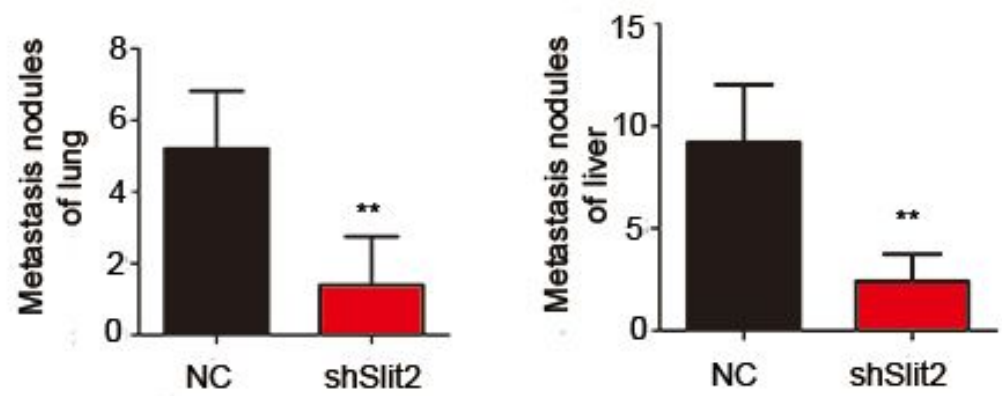

Liver

e

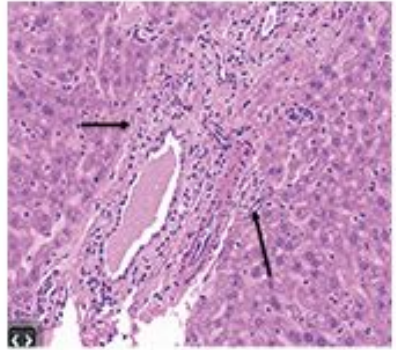

NC

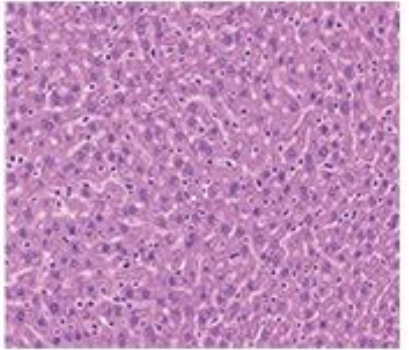

shSlit2

Figure 6

Slit2 enhances NPC metastasis in vivo. a. Knockdown Slit2 suppress CNE1/IR cells metastasis to lung and liver in vivo as shown by bioluminescence imaging after injection of CNE1/IR and CNE1-IR/shSlit2 cells for 5 weeks. b: The number of lung metastatic nodules were counted.c: The number of liver metastatic nodules were counted. $d$ : Representative lung tissue sections from each group were shown (H\&E stain; magnification, $\times 100$ ). Black arrows indicate lung metastasis.e: Representative liver tissue sections from each group were shown (H\&E stain; magnification, $\times 100)$. Black arrows indicate liver metastasis. *, $\mathrm{P}<0.05 ; * \star, \mathrm{P}<0.01$ 